\title{
Factores determinantes de la cesárea en los hospitales de Tumbes (2011)
}

\section{Decisive factors of the caesarean operation in the hospitals of Tumbes (2011)}

\author{
Gladys Patiño*
}

http://dx.doi.org/10.21503/CienciayDesarrollo.2012.v15i2.03

\section{RESUMEN}

El aumento de la tasa de cesárea en los hospitales de Tumbes es motivo de preocupación. En el hospital de apoyo I-2 José Alfredo Mendoza Olavarría, la tasa de cesárea varió entre el $35 \%$ y 49 \% durante los años 2003-2005, mientras que el hospital I EsSalud registró una tasa del $45 \%$ en el año 2006. Frente a esta realidad, se planteó el problema de investigación para determinar cuáles fueron los factores determinantes de la cesárea en los hospitales del departamento de Tumbes durante el año 2011. Con el objetivo de conocer los factores maternos y fetales asociados a la cesárea y la frecuencia de la operación, se realizó el estudio en las cesareadas de los hospitales del Ministerio de Salud y EsSalud de Tumbes durante seis meses. Las técnicas e instrumentos de recolección de datos fueron observacionales, utilizándose una ficha de recolección de datos que contenía los antecedentes personales y ginecoobstétricos de la gestante, así como datos fundamentales del recién nacido. Se realizaron entrevistas a las pacientes cesareadas, utilizando un cuestionario con preguntas estructuradas y no estructuradas, para reseñar los datos relacionados con la indicación de la cesárea. Se encontraron que en el Hospital del Ministerio de Salud, la edad promedio fue de 26,4 años, mientras que en el de EsSalud fue de 29,4. En cuanto al control prenatal, fueron gestantes controladas con más de 6 controles prenatales y con embarazos previos a quienes se les explicó el motivo pero no las probables complicaciones de la cesárea. Los recién nacidos en el hospital del Ministerio de Salud tuvieron en promedio un peso de $3194 \mathrm{~g}$ y un apgar de 9,1, mientras que en la seguridad social el peso fue de $3200 \mathrm{~g}$ y un apgar promedio de 9,2. La frecuencia de cesárea en el hospital de EsSalud fue de $58,76 \%$, y de $49,52 \%$ en el MINSA.

Palabras clave: cesárea, factores determinantes.

\section{ABSTRACT}

The increased rate of cesarean section in hospitals in Tumbes is a concern. At the support hospital I-2 Jose Alfredo Mendoza Olavarría, the cesarean rate varied between 35\% and 49\% during 2003-2005, while the EsSalud I hospital showed a rate of $45 \%$ in 2006. This is why the following problem arises: What were the determinants of caesarea at the hospitals in Tumbes in 2011? In order to meet maternal and fetal factors associated with caesarean section and the frequency of cesarean operation, it was performed the study on the cesarean at the Ministry of Health and Tumbes EsSalud hospitals for six months. The techniques and instruments for data collection were observational, using a data collection sheet containing personal history, gynecological and obstetric data of pregnant women and newborn birth. Interviews were conducted with cesarean patients, using a structured and no structured questionnaire to collect data related to the indication for caesarean section. And we were found the following results: at the Hospital 
of the Ministry of Health, the average age was 26.4 years, while at EsSalud was 29.4. In terms of prenatal care, they were pregnant women monitored over six prenatal checks up and with previous pregnancies, ones who were explained with the reason but not the likely complications of cesarean. Infants at the Hospital of the Ministry of Health had an average weight of 3194 grams and an apgar score of 9.1, while at the social security the weight was $3200 \mathrm{~g}$ and apgar average of 9.2. The frequency of caesarean section at EsSalud hospital was $58.76 \%$ and $49.52 \%$ at MINSA

Keywords: cesarean, determinants.

\section{INTRODUCCIÓN}

El curso delos embarazos ysu culminación natural fueron históricamente eventos sociales que incorporaban a la familia y a su comunidad, sin ninguna o con muy poca participación médica, pues su desarrollo resultaba normal en más del $90 \%$ de las veces. ${ }^{1}$ Hoy son sucesos más médicos que sociales, una mutación que ha transformado paulatinamente en pacientes a las mujeres embarazadas y a las parturientas normales. Este proceso de creciente medicalización, a veces excesivo, ha producido efectos no siempre deseables, entre los que se destaca el aumento dramático en las tasas de operación cesárea, que de un moderado $5 \%$ en la década de 1960, en Puerto Rico, llegó a oscilar entre $29 \%$ para 1985 , mientras que en Holanda era de $6,5 \%{ }^{1,2}$.

Dentro de las recomendaciones para la apropiada tecnología del nacimiento, señaladas por la Organización Mundial de la Salud (OMS), se establece que "no existe justificación en ninguna región geográfica específica para tener más de un 10 a 15\% de cesáreas, ya que no existen evidencias de que se requiera una operación cesárea después de una cesárea transversa segmentaria inferior. Se deberían favorecer los partos vaginales en cesareadas anteriores, en lugares donde existan las facilidades quirúrgicas en caso de emergencia”.

Fernando José (Perú) encontró una tasa de cesárea de 25,7 \%, mientras que el 95,4\% de las pacientes cesareadas tuvo una gestación con feto único. El 71,5\% de los casos tuvo control prenatal adecuado. El 56,9 \% de los casos tuvo edades entre 20 y 35 años, 32, $1 \%$ fueron menores de 20 años, y 11\% mayores de 35 años. Las principales indicaciones para el parto por cesárea fueron: desproporción céfalo-pélvica $(21,1 \%)$, sufrimiento fetal agudo (20,2\%), cesárea previa $(11,9 \%)$ y mala presentación fetal $(10,1 \%)$.

El objetivo principal del estudio fue determinar los factores asociados a la cesárea en los hospitales de Tumbes durante el 2011. Esta información permitió hacer un análisis interno de la cesárea como aporte necesario para el proceso de autocrítica que conlleve a una adecuada prescripción de la cesárea en la atención de la gestante, formulando recomendaciones precisas a través de estrategias de intervención que puedan ser reproducidas para elevar la eficiencia de la gestión de los servicios de salud, sobre todo los materno-perinatales.

\section{MATERIAL Y MÉTODOS}

El diseño de la investigación fue descriptivo, prospectivo y longitudinal. La población estudiada estuvo compuesta por la totalidad de gestantes a las que se les practicó la cesárea en el Hospital de Apoyo "JAMO", del Ministerio de Salud, y el Hospital de Nivel I "Carlos Cortés Jiménez”, de EsSalud, durante el período de seis meses, de junio a diciembre del 2011. La muestra estuvo conformada en ambos casos por 30 pacientes cesareadas de los hospitales MINSA y EsSalud. Los instrumentos utilizados fueron la ficha de recolección de datos y la encuesta. 
Recogidos los datos, se realizó una crítica de la información y se presentaron en cuadros y gráficos estadísticos. Para tal efecto, se utilizó el paquete estadístico SPSS. El estadístico utilizado fue la medida de tendencia central (media aritmética).

\section{RESULTADOS}

En este capítulo se presentan los resultados a manera de tablas.

Tabla 1. Diagnóstico de las cesáreas en Hospital MINSA Tumbes, 2011

\begin{tabular}{lcc} 
Causa de cesárea & Frecuencia & $\%$ \\
Cesareada anterior & 6 & 20,0 \\
\hline Cesareada anterior + Enfermedad hipertensiva embarazo & 4 & 13,3 \\
Cesareada anterior + Presentación alta & 2 & 6,7 \\
\hline Distocia de presentación & 2 & 6,7 \\
\hline Estrechez pélvica & 2 & 6,7 \\
\hline Estrechez pélvica + Cesareada anterior & 4 & 13,3 \\
Incompatibilidad céfalo-pélvica & 2 & 6,7 \\
\hline Macrosomía fetal & 4 & 13,3 \\
\hline Presentación podálica & 2 & 6,7 \\
\hline Trabajo de parto disfuncional & 2 & 6,7 \\
Total & 30 & 100,0 \\
\hline
\end{tabular}

Tabla 2. Diagnóstico de las Cesáreas en Hospital EsSalud Tumbes 2011

\begin{tabular}{lcc} 
Causa del parto & Frecuencia & $\%$ \\
Cesareada Anterior + Presentación pélvica & 3 & 10,0 \\
\hline Desproporción céfalo-pélvica & 3 & 10,0 \\
Presentación alta & 6 & 20,0 \\
\hline Cesareada anterior & 6 & 20,0 \\
\hline Incompatibilidad céfalo-pélvica & 3 & 10,0 \\
Ruptura prematura de membranas & 3 & 10,0 \\
Hipertensión gestacional & 3 & 10,0 \\
Parto prematuro. Hemorragia del tercer trimestre & 3 & 10,0 \\
\multicolumn{1}{r}{ Total } & 30 & 100,0 \\
\hline
\end{tabular}


Tabla 3. Control prenatal en cesareadas del Hospital Minsa Tumbes, 2011

\begin{tabular}{ccc}
$\mathrm{N}^{\circ}$ controles & Frecuencia & $\%$ \\
5 & 4 & 13,3 \\
6 & 4 & 13,3 \\
7 & 6 & 20,0 \\
8 & 6 & 20,0 \\
9 & 4 & 13,3 \\
10 & 4 & 13,3 \\
11 & 2 & 6,7 \\
\hline Total & 30 & 100,0 \\
\hline
\end{tabular}

Tabla 4. Control prenatal en cesareadas del Hospital EsSalud Tumbes, 2011

\begin{tabular}{ccc}
$\mathrm{N}^{\circ}$ controles pre natal & Frecuencia & $\%$ \\
4 & 6 & 20,0 \\
5 & 6 & 20,0 \\
6 & 6 & 20,0 \\
7 & 9 & 30,0 \\
10 & 3 & 10,0 \\
\hline Total & 30 & 100,0 \\
\hline
\end{tabular}

Tabla 5. Frecuencia de cesárea en hospitales de Tumbes, 2011

\begin{tabular}{ccccc} 
Tipo de parto & \multicolumn{2}{c}{ HOSPITAL MINSA } & \multicolumn{2}{c}{ HOSPITAL EsSalud } \\
& $\mathrm{n}$ & $\%$ & & $\mathrm{n} \%$ \\
\\
Cesárea & 1446 & 49,52 & 409 & 58,76 \\
\hline Vaginal & 1474 & 50,48 & 287 & 41,24 \\
\hline Total & 2920 & 100,0 & 696 & 100,00 \\
\hline
\end{tabular}

\section{DISCUSIÓN}

Sobre las causas de las cesáreas realizadas a las pacientes (Tabla 1 y Tabla 2), estas se presentaron en orden de importancia. En el MINSA: por ser cesareada anterior, $20 \%$; por enfermedad hipertensiva del embarazo, $13,3 \%$; por macrosomía fetal, $13,3 \%$, y por presentación alta, entre otras, 6,7\%. En EsSalud, la presentación alta mostró un 20\%, la cesareada anterior, $10 \%$, y el resto, otras causas.

Nelly Laguado (España, 2010) refiere que el factor materno más frecuente fue la cesárea electiva; como factor fetal, la evidencia de infección intrauterina (toxoplasmosis) fue el 
factor fetal más encontrado. Patricia Pavón y colaboradores (México, 2002) en su estudio encontraron que los indicaciones más frecuentes fueron sufrimiento fetal agudo (28,4 \%), desproporción céfalo-pélvica $(22,4 \%)$ y cesárea previa $(15,2 \%)$.

El Instituto Mexicano del Seguro Social (2004) reportó un estudio para determinar causas y tendencias de la operación cesárea. Las causas más frecuentes fueron cesárea previa $(35,3 \%)$ y desproporción céfalo-pélvica (20,5\%). Para Emerson, en República Dominicana, la causa más frecuente fue la cesárea anterior (iterativa) (33,6 $\%)$, seguida por la desproporción céfalo-pélvica $(22,1 \%)$. Alberto Gonzalo Romero (Argentina), para conocer la incidencia y las indicaciones de cesáreas, encontró las siguientes causas: cesárea iterativa: 249 (22,5\%), sufrimiento fetal agudo: 148 (13,5\%), cesárea anterior: 120 (11 $\%)$, presentación podálica: 111 (10\%), y gestación múltiple: 82 (7,5\%). José Fernando Echáis (Perú) reporta las principales indicaciones para cesárea: desproporción céfalo-pélvica (21,1\%), sufrimiento fetal agudo $(20,2 \%)$, cesárea previa $(11,9 \%)$ y presentación podálica $(9,2 \%)$. Es evidente que en Tumbes la cesárea anterior es la principal causa, por ser frecuente la tendencia a no dar la oportunidad del parto vaginal en forma general, y sin considerar el período intergenésico, se piensa más en los riesgos y no en las complicaciones derivadas de una segunda intervención.

En relación al control prenatal, en el MINSA se encontró que un $40 \%$ tuvo entre 7 y 8 controles; en EsSalud, un 50 \% tuvo entre 6 y 7 controles. María Eugenia Garza Elizondo, en su estudio "Patrón de uso de los servicios de atención prenatal, parto y puerperio", realizado en la Seguridad Social Mexicana, reporta que en las mujeres cuyo embarazo culminó en parto o cesárea, el promedio de consultas prenatales de las mismas fue de $6,2 \pm 4,1$. Carmen Soto realizó en Chile el trabajo "Educación prenatal y su relación con el tipo de parto: una vía hacia el parto natural", donde aplicó una intervención educativa durante la gestación y observó los efectos sobre el embarazo, parto y recién nacido, concluyendo que la educación en el control prenatal utilizando protocolos reduce el número de intervenciones rutinarias e innecesarias durante el embarazo y parto. En Tumbes, llama la atención que, a pesar de tener un promedio aceptable de controles prenatales en los que se debería evaluar la posibilidad del parto vaginal, en el último momento se decida la intervención cesárea. Es en este aspecto donde se requiere una mayor intervención y educación de las gestantes para que puedan ser parte de la decisión por la vía del parto con una preparación previa e interconsultas frecuentes con Ginecología. Carmen Soto y colaboradores (Chile, 2006) realizaron un estudio cuyo objetivo fue aplicar una intervención educativa durante la gestación y observar los efectos sobre el embarazo, parto y recién nacido. Concluyeron que la educación en el control prenatal utilizando protocolos reduce el número de intervenciones rutinarias e innecesarias durante el embarazo y parto. Igualmente, en Chile se realizó una intervención educativa durante la gestación sobre nulíparas, basándose en el hecho de que la principal causa de cesárea es haber tenido una previa, y por tanto, la estrategia debe dirigirse a evitar la primera cesárea. La principal conclusión interesante para este artículo es que la tasa de cesáreas en el grupo de estudio fue del $7,5 \%$, frente al $27,3 \%$ del de referencia, y que las causas que determinaron las cesáreas responden a las mismas causas que reporta la literatura.

Por otra parte, es lógico pensar que la primera intervención debe realizarse durante la educación maternal, permitiendo a la mujer embarazada formarse una opinión con tiempo suficiente para tomar decisiones. No obstante, "en la mayoría de las clases de preparación paras el parto, se evita hablar de las circunstancias que rodean la práctica de una cesárea, con la excusa de no causar preocupación a las embarazadas". De esta afirmación 
puede deducirse que se plantean dudas acerca de la información que recibe la mujer durante las clases preparto, y que si esto se confirma, no es el mejor camino para conseguir el objetivo deseado.

En Tumbes, la información que se brinda no es uniforme, y es necesaria mayor información, educación en las gestantes y en la pareja o los familiares para que conozcan las probables complicaciones y riesgos de la intervención cesárea, a fin de que puedan tomar una decisión consensuada, considerando el principio ético de autonomía.

La frecuencia de cesárea en el MINSA fue de 49,52 \%, y en EsSalud, 58,76 \%. Esteban Puentes-Rosas y colaboradores estudiaron el comportamiento de la cesárea durante el periodo de diez años (1994 a 2003) en instituciones públicas $y$ privadas, y encontraron que fue ligeramente superior a $35 \%$, cifra menor que la que se presenta en nuestro estudio. Las instituciones de la Seguridad Social presentaron un porcentaje de cesáreas de 38,2 \%. Patricia Pavón y colaboradores (México), en su estudio para determinar la frecuencia y las indicaciones más comunes de la cesárea, reportan como resultados una frecuencia de cesáreas de $36,7 \%$, mientras que Fernando José (2004) en su estudio encontró una tasa de cesáreas de $25,7 \%$.

La diferencia entre los dos centros asistenciales se atribuye a que en EsSalud los ginecólogos acuden al llamado de retén, en tanto que en el MINSA forman parte del Equipo Básico de Guardia, a pesar de que el Hospital de la Seguridad Social cuenta con cuatro ginecólogos de planta y en el MINSA solamente con dos especialistas, pero con un mayor número de obstetrices. Aun así, las cifras son extraordinariamente altas para tratarse de hospitales de primer nivel de atención, y tienden a aumentar si no se toman medidas, o se realizan acciones de intervención, sobre todo en la indicación de la cesárea por el hecho de ser cesareada anterior o la falta de preparación psico- profiláctica y una monitorización adecuada del trabajo de parto que permita la vía vaginal.

Es indudable que el número de cesáreas es elevado, que la presión social tiene parte de responsabilidad y que no hay una conciencia colectiva y social de los riesgos que representa una cesárea. Habría que intervenir proporcionando la información necesaria - de forma adecuada y basada en evidencias- a la mujer embarazada para que esté preparada con tiempo, y pueda participar en las decisiones que afecten su parto con conocimiento y libertad, interviniendo sobre las familias y la sociedad para crear esa conciencia colectiva favorecedora de nacimientos por vía natural, disminuyendo así el número de cesáreas. La mujer debe ser protagonista de su propio parto, y por ello, participar de todas las decisiones que la afecten, teniendo la información y libertad para decidir por sí misma.

\section{CONCLUSIONES}

1. Los factores maternos para la indicación de cesárea en los dos hospitales de Tumbes estudiados durante el 2011 fueron las cesáreas anteriores en las pacientes mayores de 25 años, con una gestación previa y más de seis controles prenatales.

2. Se cumplió con informar a todas las pacientes el motivo de las cesáreas, pero no las probables complicaciones.

3. La frecuencia de cesáreas fue muy alta en los dos hospitales de Tumbes, y mucho mayor en el hospital Carlos Cortés Jiménez de EsSalud (58,7 $\%)$, en comparación con el hospital José Alfredo Mendoza Olavarría del MINSA (49,52\%).

4. Se comprueba que los factores asociados a la cesárea son fundamentalmente maternos.

\section{REFERENCIAS BIBLIOGRÁFICAS}

1. Amu, O.; Rajendran, S; Bolaji, I. "Maternal Choice Alone Should not Determine 
Method of Delivery". BMJ. 1998; 317:462465.

2. ESSALUD. Oficina de Estadística. "Reporte de indicadores de salud reproductiva en Tumbes". Boletin 2006. EsSalud Tumbes.

3. Familia López, E. Indicaciones de cesáreas. UNMSM. Oficina General del Sistema de Bibliotecas y Biblioteca Central, 2008

4. Fernando Echáis, J. "Cesárea, experiencia en el Hospital General de Oxapampa”. Revista Ginecol. Obstet. 2004; 50 (2): 106-110.

5. Gonzalo-Romero, A. Incidencia y principales indicaciones de cesáreas. Corrientes, Argentina, 2003

6. González, W. Situación de la salud materna y perinatal en Tumbes. Informe de Gestión 2006. Dirección Regional de Salud Tumbes.

7. Instituto Mexicano del Seguro Social. "Operación cesárea: estudio de causas y tendencias en un hospital de segundo nivel". Revista médica del IMSS. 2004; 42(3)

8. Patiño, G. Frecuencia e indicaciones de operación cesárea en el Hospital I-IPSS Tumbes. Tesis para optar el título de Licenciada en Obstetricia. Universidad Nacional de Tumbes, Facultad de Ciencias de la Salud, 1994.

9. Pavon, P.; Golascoechea, M. C.; Ramírez, M., Landa, V. "Frecuencia e indicaciones de la operación cesárea en el Hospital Escuela de la Universidad Veracruzana”. Rev. Méd. UV. México. 2002; 2(2): 13-18.
10. Puentes-Rosas, E.; Gómez-Dantés, O., Garrido-La Torre, F. "Las cesáreas en México: tendencias, niveles y factores asociados". Rev. Salud Pública. Méx. Cuernavaca. 2004; 46(1).

11. Soto, C.; Teuber, H.; Cabrera, C.; Marín, M.; Cabrera, J.; Da Costa, M.; Araneda. "Educación prenatal y su relación con el tipo de parto: una vía hacia el parto natural". Rev. Chil. Obstet. Ginecol. Santiago. 2006; 71(2).

12. Uzcátegui, O.; Cabrera, C. "Aspectos bioéticos de la cesárea y consentimiento informado". Rev. Obstet. Ginecol. Venez. 2002

13. Zapata, J. Morbi-mortalidad materno perinatal en adolescentes y adultas jóvenes atendidas en el Hospital de Apoyo No 01 "José A. Mendoza Olavarria" de Tumbes durante los Años 1994-1998. Tesis para optar el Título de Licenciado en Obstetricia. Universidad Nacional de Tumbes, 1999. 